\title{
Burned-Out Testicular Cancer: Really a Different History?
}

\author{
Claudia Mosillo Simone Scagnoli Giulia Pomati \\ Salvatore Caponnetto Maria Laura Mancini Mario Bezzi \\ Enrico Cortesi Alain Gelibter \\ Policlinico Umberto I, Rome, Italy
}

\section{Keywords}

Testicular cancer - Burned-out phenomenon · Germ cell tumor · Chemotherapy · Positron emission tomography

\begin{abstract}
Two or more histological types characterize more than $60 \%$ of testicular germ cell tumors (GCTs). Burned-out testicular tumor refers to partial or complete histological regression of the primary testicular lesions. The most frequent GCT type involved in this kind of histological regression is choriocarcinoma, followed by embryonal carcinoma. To our knowledge, there are no cases of the burned-out phenomenon in teratoma. We report a case of a 19year-old man presenting to our institute with a right testicular lesion, evidence of mediastinal and abdominal lymph node metastasis, and high levels of GCT serum biomarkers. After orchiectomy, the histopathological examination showed a mixed GCT: mature teratoma, immature teratoma, and histological features of testicular cancer regression (burned-out phenomenon). The patient underwent first-line chemotherapy (BEP regimen) which resulted in a complete instrumental and biochemical response after 4 cycles. Teratoma is considered a less aggressive type of GCT. In this particular case, metastatic disease seems to result from nongerm cell components which underwent early spontaneous regression.

(C) 2017 The Author(s)

Published by S. Karger AG, Basel
\end{abstract}




\section{Background}

More than $90 \%$ of testicular tumors originate from germ cells, which are the main cell population of the testis. The peak of the incidence of testicular germ cell tumors (GCTs) occurs between the ages of 15 and 35 years. The risk factors for developing testicular cancer include cryptorchidism, Klinefelter syndrome, a family history of testicular tumors, a prior history of a testicular tumor, infertility, and sperm abnormalities [1-3].

Sixty percent of GCTs are mixed, characterized by two or more histological types. The most frequent combinations are: teratoma, embryonal carcinoma, and yolk sac tumor; seminoma and embryonal carcinoma; and teratoma and embryonal carcinoma. The presence of aggressive histotypes worsens the prognosis [1].

A burned-out testicular tumor refers to the presence of a metastatic extragonadal GCT with histological regression of the primary testicular lesion [4, 5]. Histological features that are helpful to define a diagnosis of a burned-out testicular GCT include scar formation, intratubular calcifications, lymphoplasmacytic infiltrate, hemosiderin-containing macrophages, and testicular atrophy [6].

Choriocarcinoma is most likely to burn out, followed by embryonal carcinoma; regression is uncommon in testicular seminoma and probably does not occur in teratoma [4]. We report a case with partial burned-out testicular mixed GCT that metastasized to the retroperitoneal and mediastinal area.

\section{Case Report}

A 19-year-old man complained about gynecomastia and the presence of a testicular lesion. In his recent pathological history the patient reported testicular inflammation 1 year earlier. Physical examination revealed a minimum increase of breast tissue bilaterally and centimetric mass in the right testis. The left testis was found to be normal.

Scrotal ultrasound revealed a solid testicular mass $(18 \times 13 \mathrm{~mm})$ with nonhomogeneous ecotexture and calcifications. Abdominal and thorax computed tomography (CT) (Fig. 1) revealed bulky abdominal and mediastinal lymphadenopathies (maximum diameter $44 \mathrm{~mm}$ ).

High levels of lactate dehydrogenase (LDH) $835 \mathrm{mg} / \mathrm{dL}$ (normally $260-550 \mathrm{mg} / \mathrm{dL}$ ) and $\alpha$-fetoprotein (AFP) $306 \mathrm{ng} / \mathrm{mL}$ (normally $<10 \mathrm{ng} / \mathrm{mL}$ ) were found in the blood tests, while beta-human chorionic gonadotrophin hormone (b-hCG) $0.5 \mathrm{mIU} / \mathrm{mL}$ (normally $<4 \mathrm{mlU} / \mathrm{mL}$ ) was within normal limits.

On September 22, 2016, the patient underwent right orchiectomy, and histological specimens revealed testicular GCTs of $17 \mathrm{~mm}$ composed of mature teratoma (85\%) and immature teratoma (15\%). There was no other histological type of tumor cells, lymphovascular and rete testis invasion, pT1. The histopathological examination showed interstitial fibrosis with calcifications, hemosiderin depositions, and inflammatory cell infiltrate in the peritumoral area, indicative of partial regressed malignancy ("burnedout" phenomenon). The tissue around these aggregates was characterized by intratubular germ cell neoplasia and atrophy of the seminiferous tubules.

The patient arrived at our division after surgery. He had a good performance status and we repeated postoperative serum tumor markers that revealed LDH $932 \mathrm{mg} / \mathrm{dL}$, AFP $803 \mathrm{ng} / \mathrm{mL}$, and b-hCG $1 \mathrm{mIU} / \mathrm{mL}$.

Due to the bulky and diffuse lymph node disease revealed at the preoperative CT body scan we decided to immediately start with first-line chemotherapy. A combination treatment with PEB (bleomycin, etoposide, and cisplatin) was given every 3 week for 4 courses.

After the first 2 courses, serum tumor markers dramatically decreased (LDH $203 \mathrm{mg} / \mathrm{dL}$, AFP $7 \mathrm{ng} / \mathrm{mL}$, and b-hCG $0.5 \mathrm{mIU} / \mathrm{mL}$ ) and 1 month after the end of the 4th course, the patient underwent a positron emission tomography (PET) scan.

The PET/CT scan (Fig. 2) showed dimensional reduction of abdominal and mediastinal lymphadenopathy (wider axis reduced from 44 to $34 \mathrm{~mm}$ ), without pathological uptake in all the sites of disease. Similarly the values for AFP and others tumor markers returned to within normal limits. 
After 3 months, the patient's repeated CT scan revealed further shrinkage of parameter lesions with extensive central necrosis. Surgical evaluation in the multidisciplinary unit was performed with no indication for surgery due to technical challenges. The patient underwent in laboratory and instrumental followup.

\section{Discussion}

About $60 \%$ of testicular mixed GCT appears with advanced disease (stage II or III). Early metastases most commonly occur in the retroperitoneal lymph nodes and progressively can involve mediastinal and supraclavicular lymph nodes $[1,2]$.

Infrequently, testicular GCT can regress spontaneously after its metastatic spread to the lymph nodes. This phenomenon is called "burned-out." Microscopically, testicular GCT can be completely or partially burned out [4].

Spontaneous regression of testicular GCT has been reported in several cases. Prym [7] described the first case of burned-out tumor characterized by a fibrous scar with no tumor in the testis in a man presenting with disseminated choriocarcinoma.

Azzopardi et al. [6] subsequently reported 17 patients with widespread embryonic and choriocarcinoma with clinically normal testis. In all instances, a fibrous scar was found in the testis; in 13 cases amorphous hematoxylin-staining deposits were observed in dilated seminiferous tubules and half of the scars contained an infiltrate of hemosiderin-laden macrophages, which presumably represents the destruction of the original tumor.

Teratoma is considered less aggressive and less likely to metastasize than other nonseminomatous GCT, and it frequently contains elements which do not produce serum tumor markers (AFP and HCG) [8].

Heidenreich et al. [9] reported their experience with 44 cases of primary pure testicular teratoma; histopathological evaluation of the primary tumor specimen demonstrated scars and calcifications indicating a burned-out tumor in 16 of 20 patients (80\%) with metastatic disease.

There are different unconfirmed theories to explain the burned-out phenomenon that led to a spontaneous regression of a primary GCT after metastasis spreading to different sites. The mechanism of this regression has yet to be elucidated. A possible explanation is an important immune response stimulated by the host or, otherwise, an ischemia caused by the high metabolic rate of the neoplasm outgrowing its blood supply.

In this case report, according to the literature presented, lymph-node diffuse metastases in testicular mature teratoma seem to result from aggressive germ cell components which have undergone early regression.

In our case, this hypothesis cannot be confirmed by histological evaluation. On the other hand, the high response to chemotherapy, the normalization of specific serum markers, and the low rate of metastasizing teratoma support our hypothesis.

\section{Statement of Ethics}

The authors have no ethical conflicts to disclose. 


\section{Case Reports in Oncology}

\section{Disclosure Statement}

The authors have no conflicts of interest to declare.

\section{References}

1 Bosl GJ, Motzer NJ: Testicular germ-cell cancer. N Engl J Med 1997;337:242.

2 Surveillance, Epidemiology and End Result Program (SEER): testis cancer. National Cancer Institute, 2015. http://seer.cancer.gov/statfacts/html/testis.html.

3 Looijenga LH, Oosterhuis JW: Pathogenesis of testicular germ cell tumours. Rev Reprod 1999;4:90

-4 Calvo OA, Rodríguez Alonso A, Pérez García D, Domínguez Freire F, Alonso Rodrigo A, Rodríguez Iglesias B, Benavente Delgado J, Barros Rodríguez JM, Fiaño Valverde C, Nogueira March JL: Extragonadal germ cell tumour with 'burned-out' phenomenon in the testis. Actas Urol Esp 1999;23:880-884.

-5 Fabre E, Jira H, Izard V, Ferlicot S, Hammoudi Y, Theodore C, Di Palma M, Benoit G, Droupy S: "Burnedout" primary testicular cancer. BJU Int 2004;94:74-78.

6 6 Azzopardi JG, Mostofi FK, Theiss EA: Lesions of testes observed in certain patients with widespread choriocarcinoma and related tumors: the significance and genesis of hematoxylin-staining bodies in human testis. Am J Pathol 1961;38:207-225.

7 Prym P: Spontanheilung eines bösartigen, wahrscheinlich chorionephiteliomatösen Gewächses im Hoden. Virchows Arch Pathol Anat 1927;265:239-258.

8 Germa-Lluch JR, Begent RH, Bagshawe KD: Tumor marker levels and prognosis in malignant teratoma of the testis. Br J Urol 1980;42:850-855.

-9 Heidenreich A, Moul JW, McLeod DG, Mostofi FK, Engelmann UH: The role of retroperitoneal lymphadenectomy in mature teratoma of the testis. J Urol 1997;157:160-163.

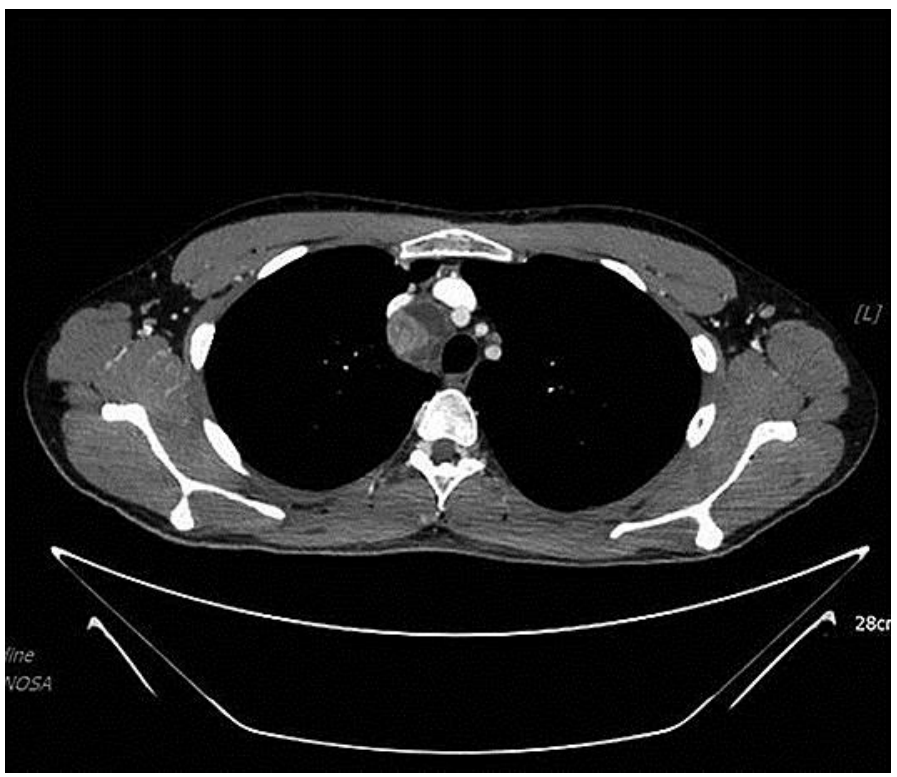

Fig. 1. CT image obtained before treatment. 


\section{Case Reports in Oncology}
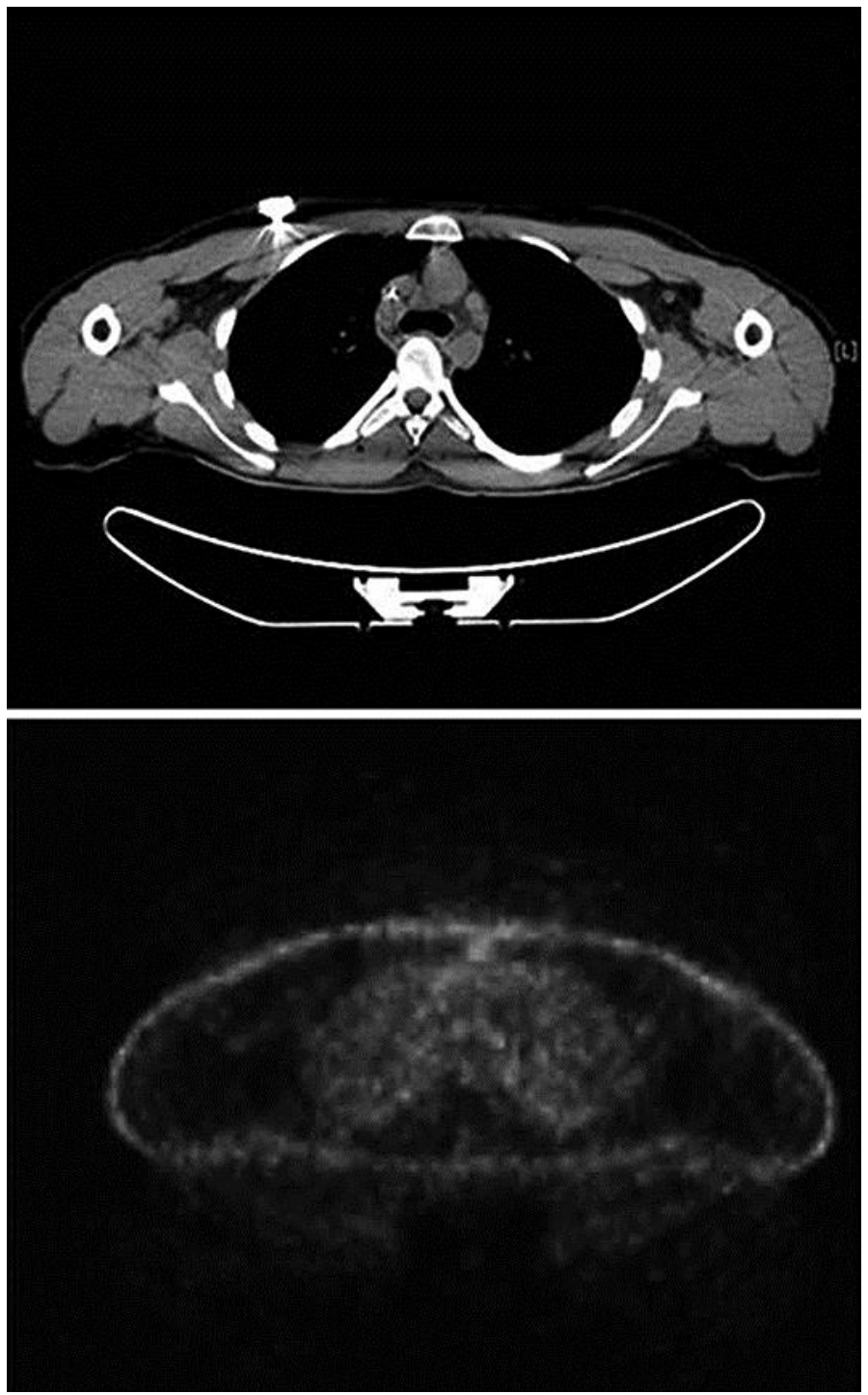

Fig. 2. CT and PET images obtained after chemotherapy. 